\title{
Exploring the Link between Helicobacter pylori Eradication and Metachronous Gastric Cancer Development
}

\author{
Hyo-Joon Yang \\ Division of Gastroenterology, Department of Internal Medicine and Gastrointestinal Cancer Center, Kangbuk Samsung Hospital, Sungkyunkwan \\ University School of Medicine, Seoul, Korea
}

See "Long-Term Effects of Helicobacter pylori Eradication on Metachronous Gastric Cancer Development" by Seung Jun Han, et al. on page 133, Vol. 12. No. 2, 2018

Endoscopic resection has been accepted as an alternative to gastrectomy for early gastric cancer (EGC), providing comparable survival rates and a better quality of life. ${ }^{1}$ Preserving most of the non-cancerous mucosa, however, resulted in the development of metachronous gastric cancer (MGC) in $>20 \%$ of the patients during the 10 years after endoscopic resection. ${ }^{1} \mathrm{He}-$ licobacter pylori eradication is recommended by various guidelines and consensus reports after endoscopic resection of EGCs. Indeed, it is the only available intervention that can possibly reduce the risk for MGCs. However, the results from randomized controlled trials regarding whether the eradication of $H$. pylori can reduce the development of MGCs are still conflicting. ${ }^{2,3}$ Furthermore, there is little information on the effect of $H$. pylori eradication on premalignant conditions, such as gastric mucosal atrophy and intestinal metaplasia, in a group of people with a high risk of gastric cancer.

In this issue of Gut and Liver, Han et al. ${ }^{4}$ retrospectively followed 565 patients who had undergone endoscopic resection of EGC between 2005 and 2011 for a median of 60 months. The patients were divided into the $H$. pylori-negative $(n=157)$, -eradicated $(n=212)$, and -persistent $(n=196)$ groups and then followed for the changes in atrophic gastritis and intestinal metaplasia and the development of MGCs. Histologic grades of corpus atrophy were similar between the $H$. pylori-eradicated and -persistent groups at baseline (1.06 and 1.15, respectively, $p>0.05$ ), but corpus atrophy was significantly less severe in the eradicated group than in the persistent group at follow-up (1.03 and 1.29 , respectively, $\mathrm{p}=0.029$ ). The $H$. pylori negative group showed a constantly less severe corpus atrophy at baseline and follow-up than both the $H$. pylori-eradicated and -persistent groups (all $\mathrm{p}<0.05$ ). There were no noticeable differences or changes in the antrum atrophy or intestinal metaplasia. A total of 50 patients developed MGCs during the follow-up period (50/565, 8.8\%): 20 in the negative group (20/157, 12.7\%), 12 in the eradicated group (12/212, 5.7\%), and 18 in the persistent group (18/196, 9.2\%). The difference in the cumulative risk of developing MGCs between $H$. pylori-eradicated and -persistent groups was only marginally significant in the overall cohort ( $p=0.059$ by log-rank test) and statistically significant in the subgroup of patients younger than 70 years ( $\mathrm{p}=0.018$ by logrank test). The risk for MGCs was the highest in the $H$. pylorinegative group.

The following interpretations can be made. First, $H$. pylori eradication could delay the progression of corpus atrophy even in patients who have already developed gastric cancer but not the progression of antral atrophy or intestinal metaplasia. Gastric atrophy and intestinal metaplasia may not be reversed in these patients, unlike that suggested by the results from the previous studies in cancer-naïve people. ${ }^{5}$ Second, $H$. pylori eradication may reduce the risk of developing MGCs, and the effect would be more prominent in patients younger than 70 years. These results augment the current evidence favoring $H$. pylori eradication after endoscopic resection of EGC. Therefore, $H$. pylori eradication would be the most beneficial among the patients with EGC who are younger than 70 years and with no or low grades of corpus atrophy because it could not reverse atrophy but only slows the progression.

The authors suggested delayed progression of corpus atrophy

Correspondence to: Hyo-Joon Yang

Division of Gastroenterology, Department of Internal Medicine and Gastrointestinal Cancer Center, Kangbuk Samsung Hospital, Sungkyunkwan University School of Medicine, 29 Saemunan-ro, Jongno-gu, Seoul 03181, Korea

Tel: +82-2-2001-8330, Fax: +82-2-2001-8360, E-mail: hyojoonyang@gmail.com pISSN 1976-2283 eISSN 2005-1212 https://doi.org/10.5009/gnl17493

@) This is an Open Access article distributed under the terms of the Creative Commons Attribution Non-Commercial License (http://creativecommons.org/licenses/by-nc/4.0) which permits unrestricted non-commercial use, distribution, and reproduction in any medium, provided the original work is properly cited. 
as a possible link between $H$. pylori eradication and the reduced incidence of MGCs. However, it was not shown whether the corpus atrophy was associated with the development of MGCs in the analysis. Actually, only age, not atrophy or $H$. pylori infection, was independent risk factor for developing MGC in the adjusted analysis. Moreover, although $H$. pylori eradication was associated with lower grades of corpus atrophy, it was not associated with the grades of intestinal metaplasia. How did the progression of corpus atrophy affect the development of MGC without considerable progression of metaplasia? First, it is possible that the histologic grade of intestinal metaplasia was not a proper measure to reflect the changes posed by $H$. pylori eradication. It was suggested that the distribution of intestinal metaplasia could be a reliable predictor for the risk of gastric cancer. ${ }^{6}$ Second, gastric atrophy may play a crucial role in the gastric carcinogenesis, whereas intestinal metaplasia might be a mere bystander. Theories were suggested that mucosal atrophy may offer the homing to cancer stem cells in $H$. pylori-associated gastric cancer. ${ }^{7}$ Third, it is also possible that delayed progression of atrophy may not be related to the decreased risk for MGCs after $H$. pylori eradication. The two findings could only have coincided and another mechanism, such as promoter methylation of tumor suppressor genes, could have played a role in metachronous recurrence. ${ }^{8}$

It is also noticeable that the $H$. pylori-negative group showed the highest incidence of MGC despite the lowest grades of corpus atrophy, which also raises questions about the association between corpus atrophy and the risk for MGC. Another retrospective cohort study showed a lower risk for MGC among $H$. pylori-negative patients with EGC. ${ }^{9}$ Heterogeneity in the definitions of $H$. pylori negativity and false negativity among the tests for $H$. pylori infection might be the reason for these conflicting results. Thus, caution is necessary during the interpretation of the results in the $H$. pylori-negative group.

In summary, this study suggests that $H$. pylori eradication may reduce the progression of corpus atrophy and the development of MGC, especially in patients younger than 70 years. This study is significant because the authors systematically and histologically evaluated the changes in precancerous lesions after $H$. pylori eradication in patients who underwent endoscopic resection of EGC. The observation that the progression of corpus atrophy is delayed by $H$. pylori eradication warrants further studies that will explore the link between $H$. pylori eradication and the development of MGC.

\section{CONFLICTS OF INTEREST}

No potential conflict of interest relevant to this article was reported.

\section{REFERENCES}

1. Yang HJ, Kim SG, Lim JH, et al. Novel risk stratification for metachronous recurrence after curative endoscopic submucosal dissection for early gastric cancer. Gastrointest Endosc 2018;87:419428

2. Choi J, Kim SG, Yoon H, et al. Eradication of Helicobacter pylori after endoscopic resection of gastric tumors does not reduce incidence of metachronous gastric carcinoma. Clin Gastroenterol Hepatol 2014;12:793-800.e1.

3. Fukase K, Kato M, Kikuchi S, et al. Effect of eradication of Helicobacter pylori on incidence of metachronous gastric carcinoma after endoscopic resection of early gastric cancer: an open-label, randomised controlled trial. Lancet 2008;372:392-397.

4. Han SJ, Kim SG, Lim JH, et al. Long-term effects of Helicobacter pylori eradication on metachronous gastric cancer development. Gut Liver 2018;12:133-141.

5. Kong YJ, Yi HG, Dai JC, Wei MX. Histological changes of gastric mucosa after Helicobacter pylori eradication: a systematic review and meta-analysis. World J Gastroenterol 2014;20:5903-5911.

6. Shichijo S, Hirata Y, Niikura R, et al. Histologic intestinal metaplasia and endoscopic atrophy are predictors of gastric cancer development after Helicobacter pylori eradication. Gastrointest Endosc 2016;84:618-624.

7. Correa P, Houghton J. Carcinogenesis of Helicobacter pylori. Gastroenterology 2007;133:659-672.

8. Shin CM, Kim N, Lee HS, et al. Changes in aberrant DNA methylation after Helicobacter pylori eradication: a long-term follow-up study. Int J Cancer 2013;133:2034-2042.

9. Jung S, Park $\mathrm{CH}$, Kim EH, et al. Preventing metachronous gastric lesions after endoscopic submucosal dissection through Helicobacter pylori eradication. J Gastroenterol Hepatol 2015;30:75-81. 